\title{
From vision to reality: a centre of excellence for Aboriginal and Torres Strait Islander primary health care
}

\section{A centre for high-quality, culturally safe primary care, research and teaching, and improved access to specialists is open for business}

ccess to medical services continues to be problematic for many Aboriginal and Torres Strait Islander people. There are barriers relating to availability, affordability, acceptability and appropriateness, and the latter two are particularly relevant in urban areas. ${ }^{1}$ National Aboriginal Community Controlled Health Organisation (NACCHO) models of care redress these barriers. ${ }^{2}$ Five years ago, we described how the Inala Indigenous Health Service (IIHS), a Queensland Government-funded primary care service in Brisbane, had adopted the key principles underpinning the NACCHO models of care and established a culturally secure health service with an associated increase in the number of Aboriginal and Torres Strait Islander patients from 12 in 1994 to over 3000 in $2008 .^{3}$ Here we report on the expansion and maturation of the IIHS into the Southern Queensland Centre of Excellence in Aboriginal and Torres Strait Islander Primary Health Care (CoE).

The CoE aims to improve access to health care and health outcomes by providing high-quality, integrated primary and secondary care, training for the next generation of health care professionals, a research agenda focusing on chronic disease and health service delivery, and outreach services to areas where access to primary care is problematic.

In 2010, the Queensland Government provided $\$ 7$ million to construct a purpose-built clinic. At the June 2013 opening, the Queensland Minister of Health announced a further \$10.5 million for Stage 2 to accommodate existing allied health personnel (a dietitian, social worker and psychologist), research team, and community engagement team. In partnership with the University of Queensland, Stage 2 will also incorporate dental care facilities.

By May 2014, we had over 10000 adult patients registered with our Inala clinic, with 3500 attending regularly (at least three visits in the past 2 years). In the 12 months before May 2014, there were 14070 general practitioner consultations at the main clinic, plus an additional 2182 consultations at our two outreach clinics. Seven visiting specialists provide between one and four clinics monthly, significantly enhancing access to specialist services. ${ }^{4}$ Exercise stress tests and echocardiograms are done at Inala, with patients who need follow-up angiograms at the local public hospital now being more likely to attend for the procedure,
Noel E Hayman

MBBS, MPH, FAFPHM Associate Professor, ${ }^{1}$ and Clinical Director $^{2}$

Deborah A Askew BAppSci, MHlthSci, PhD Associate Professor, Associate Professor,
and Research Director

and Research Director

Geoffrey K Spurling
MBBS, MPH, DTM\&H Senior Lecturer and General Practitioner ${ }^{2}$

1 University of Queensland, Brisbane, QLD.

2 Southern Queensland Centre of Excellence in Aboriginal and Torres Strait Islander Primary Health Care, Queensland Health, Brisbane, QLD.

noel.hayman@ health.qld.gov.au

doi: 10.5694/mjal4.00766

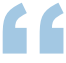

By May 2014, we had over 10000 adult patients registered with our Inala clinic possibly because of the established relationship with the cardiologist. Three new visiting specialists, a podiatrist and a physiotherapist will begin working at the $\mathrm{CoE}$ in July 2014. Patients appreciate the specialists coming to Inala rather than having to attend hospital-based specialist outpatient clinics.

The CoE research agenda has three major themes: increasing our understanding of our patients and their health status; describing and evaluating how we care for our patients; and trialling new health care delivery approaches. The Inala Community Jury for Aboriginal and Torres Strait Islander Health Research (our community research advisory group) reviews all our research to ensure the cultural appropriateness and community relevance of the topic and approach. We are cognisant of the troubled history of research with Aboriginal and Torres Strait Islander peoples ${ }^{5}$ and always try to minimise the burden of participation by using administrative data where possible - for example, computerising health check templates, enabling patient information to be stored in their medical records and, for consenting patients, in a research database. ${ }^{6}$

In addition to general practice and specialty registrars and medical and nursing students, we also provide placements for allied health students, public health undergraduate students, and Aboriginal health worker trainees. Each student or registrar is orientated to the community engagement activities of the $\mathrm{CoE}$ and to the local Aboriginal and Torres Strait Islander community with the aim of improving their understanding of urban Aboriginal and Torres Strait Islander primary health care.

We have established an expert health team that delivers culturally sensitive primary health care to Cunnamulla, Western Queensland every month. The all-Aboriginal team consists of a general practitioner, two nurses and administrative staff, accompanied at times by either an endocrinologist or a cardiologist. We also provide an outreach "Mums and Bubs" clinic in an underserviced outer suburb of Brisbane four days weekly, and a weekly clinic at a local independent girls school in Brisbane that provides secondary school education, vocational training and mentoring for students who haven't succeeded in traditional educational models.

In 2009 we wrote that new, innovative strategies were needed to improve health outcomes and life expectancy for Aboriginal and Torres Strait Islander people. ${ }^{3}$ The CoE has broken down access barriers by providing acceptable, accessible and appropriate primary-level and secondary-level care. While its governance model is not that of a community controlled health service, the CoE mirrors the community controlled sector in the philosophies and principles that inform the clinical care, community engagement, teaching, research, leadership 
and its overarching vision. In summary, the $\mathrm{CoE}$ is striving towards providing high-quality, culturally safe clinical care to underserved Indigenous communities, and making a positive contribution to the Indigenous health workforce and research base.

Competing interests: No relevant disclosures.

Provenance: Commissioned; not externally peer reviewed.

1 Scrimgeour D, Scrimgeour M. Health care access for Aboriginal and Torres Strait Islander people living in urban areas, and related research issues: a review of the literature. Cooperative Research Centre for Aboriginal Health. Darwin: Cooperative Research Centre for Aboriginal Health, 2008. https://www.lowitja.org.au/sites/default/files/docs/DP5_final-pdf.pdf (accessed May 2014)
2 National Aboriginal Community Controlled Health Organisation. Vision and principles. Our guiding principles. http://www.naccho.org.au/aboutus/vision-and-principle (accessed Jun 2014).

3 Hayman NE, White NE, Spurling GK. Improving Indigenous patients' access to mainstream health services: the Inala experience. Med J Aust 2009; 190: 604-606.

4 Spurling GK, Askew DA, Hayman NE, et al. Retinal photography for diabetic retinopathy screening in Indigenous primary health care: the Inala experience. Aust N Z J Public Health 2010; 34 Suppl 1: S30-S33.

5 Laycock A, Walker D, Harrison N, Brands J. Researching Indigenous health: a practical guide for researchers. Melbourne: The Lowitja Institute, 2011.

6 Spurling GK, Askew DA, Schluter PJ, Hayman NE. Implementing computerised Aboriginal and Torres Strait Islander health checks in primary care for clinical care and research: a process evaluation. BMC Med Inform Decis Mak 2013; 13: 108. 